\title{
Routine use of hysteroscopy before in-vitro fertilization: Systematic review and meta-analysis of randomized controlled trials
}

\author{
Doaa M Saleh', Gehad El Ashal ${ }^{1,3}$, Sherif Elsherif ${ }^{1,3}$, Sylvia Samy Awad ${ }^{1,4}$ \\ Mohammad Metwally Mahmoud ${ }^{1,4}$, Muhammad Saad Gohar ${ }^{1,4}$, Ahmed El Hadi ${ }^{1,4}$, \\ Review \\ Ahmed Elgebaly ${ }^{1,2}$ \\ Article \\ ${ }^{1}$ Medical Research Education \& Practice Association (MREP) \\ ${ }^{2}$ Faculty of Medicine, Al-Azhar University \\ ${ }^{3}$ Kasr Al Ainy School of Medicine, Cairo University, Cairo \\ ${ }^{4}$ Faculty of Medicine, Alexandria University, Alexandria, Egypt
}

\begin{abstract}
Objective: This study aimed to synthesize evidence from published Randomized Controlled Trials (RCTs) about the effectiveness of hysteroscopy in both women scheduled for first in-vitro fertilization (IVF) and women with recurrent IVF failure.

Study Design: We searched the following electronic databases: PubMed, Scopus, Web of Science and Cochrane Central. Retrieved records were screened for eligibility. Dichotomous data were pooled as relative risk (RR) in a random-effect model. We used Review Manager 5.3 for windows.

Results: Five unique RCTs with a total of 2636 patients were included. The overall effect estimates did not favour hysteroscopy group in terms of live birth from a pregnancy, ongoing pregnancy during the trial period, clinical pregnancy during the trial period, and incidence of miscarriage. The pooled analysis were not homogenous $(P<0.1)$.

Conclusion: The current evidence is insufficient to support the routine use of hysteroscopy in both women scheduled for first IVF and women with recurrent IVF failure.
\end{abstract}

Key Words: Hysteroscopy, in-vitro fertilization, meta-analysis, recurrent IVF failure

Received: $23^{\text {rd }}$ May 2019, Accepted: $2^{\text {nd }}$ August 2019

Corresponding Author: Ahmed Saber Elgebaly, Department of Obstetrics \& Gynecology, Faculty of Medicine, Al-Azhar University, Madinet Nasr, Abbassia, Cairo, Egypt, Tel.: +20 1112987286, E-mail: Ahmedelgebaly94@azhar.edu.eg

ISSN: $2090-7625$, November 2019, Vol.9, No. 4

\section{INTRODUCTION}

Infertility is a challenging medical condition that nearly affects every fifth couple at the reproductive age. In many cases, infertility can be attributed to organic conditions which require medical intervention ${ }^{[10]}$. Moreover, infertility was strongly linked to high level of psychological stress which may need a combination of different psychosocial interventions ${ }^{[18]}$. In vitro fertilization (IVF), which is a multi-stage procedure including stimulation of ovulation, oocytes retrieval, ova fertilization, culture of embryos and subsequently, the transfer of embryos to the uterine cavity, is one of the most effective treatment modalities for non-male factor infertility in the last decades ${ }^{[21]}$. IVF was reportedly resulted in born of more than 5 million livebirths since its introduction in $1970 \mathrm{~s}^{[26]}$. Despite its high cost, the success rate of IVF remains a major challenge with a large unexplained gap remains between the number of embryo transfers and the number of ongoing pregnancies ${ }^{[2,25]}$.

Implantation failure may be attributed to a variety of reasons including the embryo quality and the uterine receptivity. However, unexplained implantation failure accounts for considerable proportion of the $\operatorname{cases}^{[13]}$. The prevalenceofunsuspecteduterinepathologyinasymptomatic women that may negatively affect the uterine receptivity and reduce the chance of implantation has been reported to be as high as $50 \%{ }^{[1]}$. Therefore, a detailed examination of the intra uterine integrity prior to IVF and subsequent management of any abnormal findings is proposed as an 
effective strategy for reducing the implantation failure ${ }^{[3,12]}$. Hysteroscopy has been proposed as a minimally invasive, well tolerated, procedure that allows reliable visual assessment of the cervical canal and uterine cavity to detect any abnormality prior to $\operatorname{IVF}^{[12,24]}$. However, the current body of evidence shows a conflicting results regarding the clinical utility of routine hysteroscopy prior to $\operatorname{IVF}^{[7,19]}$.

\section{AIM OF THE WORK}

The aim of the present meta-analysis is to synthesis evidence from the published randomized controlled trials (RCTs) about the effectiveness of routine hysteroscopy in improving the pregnancy outcomes of IVF.

\section{PATIENTS AND METHODS}

We performed this review according to the preferred reporting items for systematic reviews and meta-analyses (PRISMA) statement ${ }^{[14]}$.

\section{Inclusion and Exclusion criteria:}

We included RCTs with the following criteria ; trials whose population was infertile women undergoing IVF for the first time or due to recurrent failure, the study group included women who had hysteroscopy performed prior to the IVF and the control group was those who undergoing IVF without prior hysteroscopy. In the case of multiple reports for the same study population, we analyzed data of the most complete dataset. Studies were excluded for the following reasons ; studies without reliable data for extraction, thesis and conference papers and non-English studies.

\section{Literature Search Strategy:}

We performed a comprehensive search of four electronic databases; PubMed, Scopus, Web of science and Cochrane CENTRAL using the following query (Hysteroscopy AND invitro-fertilization). We conducted an additional manual search for relevant studies in the references of included studies. Three independent reviewers screened the titles and abstracts of retrieved records, followed by full-texts screening for eligibility. Any disagreements were resolved by discussion and consensus.

\section{Data Extraction:}

The extracted data included the following domains; characteristics of study design, baseline criteria of included population, risk of bias domains and study outcomes. Four reviewers extracted the data from the included articles and any discrepancies were solved by discussion. We extracted data from graphs using PlotDigitizer software (http:// plotdigitizer.sourceforge.net/).

\section{Quality assessment:}

The quality of the retrieved RCTs was assessed according to Cochrane handbook of systematic reviews of interventions 5.1.0 (updated March 2011). Risk of bias assessment included the following domains: sequence generation (selection bias), allocation sequence concealment (selection bias), blinding of participants and personnel (performance bias), blinding of outcome assessment (detection bias), incomplete outcome data (attrition bias), selective outcome reporting (reporting bias) and other potential sources of bias. The authors' judgments are categorized as low risk, high risk or unclear risk of bias. We used the quality assessment table provided in (part 2, Chapter 8.5) the same book ${ }^{[8]}$.

\section{Measures of treatment effect:}

The primary outcomes, in studies assessed the efficacy of hyeteroscopy, were:

1- A live birth from a pregnancy which defined as delivery of a live fetus after 24 weeks of gestation.

2- Ongoing pregnancy defined as the detection of a fetal heartbeat on ultrasound at four or more weeks of gestation

3- Clinical pregnancy defined as the presence of a gestational sac four or more weeks after embryo transfer.

\section{Data Synthesis:}

Dichotomous data were pooled as relative risk (RR) in a random-effect model using inverse-variance method. We used Review Manager 5.3 for windows.

\section{Assessment of heterogeneity:}

Heterogeneity was assessed by visual inspection of the forest plots and measured by I-square and Chi-Square tests. Chi-square test was used to test the existence of significant heterogeneity while I-square quantifies the variability in effect estimates that is due to heterogeneity, if present. I-Square test was interpreted according to recommendations of Cochrane Handbook of Systematic Reviews and metaanalysis ( $0 \%$ to $40 \%$ might not be important, $30 \%$ to $60 \%$ may represent moderate heterogeneity, $50 \%$ to $90 \%$ may represent substantial heterogeneity and $75 \%$ to $100 \%$ considerable heterogeneity). Significant heterogeneity was considered at Chi-Square $P<0.1$.

\section{Sensitivity analysis:}

In order to resolve detected statistical heterogeneity, we performed sensitivity analysis excluding one study in each scenario.

\section{Publication bias:}

According to Egger andcolleagues ${ }^{[5,23]}$, publication bias assessment is not reliable for less than 10 pooled studies. 
Therefore, in the present study, we could not assess the existence of publication bias by Egger's test for funnel plot asymmetry.

\section{RESULTS}

We retrieved 664 unique citations. From which, five unique RCTs with a total of 2636 patients (Hysteroscopy group $n=1285$ and Control group $n=31351$ ) were included in the present systematic review and meta-analysis. (See PRISMA flow diagram; Figure.1).

Two included trials performed hysteroscopy to infertile women with no prior $\operatorname{IVF}^{[7,20]}$, while the remaining trials included infertile women with recurrent IVF failure ${ }^{[4,6,17]}$. Investigators involved in the embryo transfer were masked to group allocation in two included trials ${ }^{[6,20]}$ and the sample size of included studies ranged from 200 to 750 participants. Hysteroscopy and control groups did not differ significantly regarding any of baseline variables in included trials. Interestingly, Smith et al. and El-Toukhy, et al. reported that routine use of hysteroscopy does not improve live birth rates, clinical pregnancy, or ongoing pregnancy in infertile women scheduled for IVF treatment ${ }^{[6,20]}$. In contrast, the remaining three trials concluded that hysteroscopy is recommended for infertility workup before IVF even in patients with normal ultrasound finding ${ }^{[4,7,17]}$. Summary of included studies and baseline characteristics are shown in Table 1 .

The quality of the included RCTs was from moderate to high quality according to Cochrane risk of bias assessment tool. Summary of quality assessment domains of included studies is shown in Figure 2. Authors' judgments with justifications are shown in supplementary file no.1.

\section{Effect of hysteroscopy prior to IVF in pregnancy outcomes:}

The overall effect estimates did not favor hysteroscopy group in any of the following terms; a live birth from a pregnancy during the trial period (No prior IVF group' RR 1.06, 95\% CI [0.93, 1.20], $\mathrm{p}=0.41$; Recurrent IVF failure group' RR $1.29,95 \%$ CI $[0.77,2.16], p=0.33$; Figure 3 ), ongoing pregnancy during the trial period (No prior IVF group' RR $1.25,95 \%$ CI $[0.86,1.80], \mathrm{p}=0.24$; Recurrent IVF failure group' RR $0.98,95 \%$ CI $[0.82,1.19], p=0.86$; Figure 4), clinical pregnancy during the trial period (No prior IVF group' RR 1.03, 95\% CI [0.88, 1.21], p =0.68; Recurrent IVF failure group' RR $1.31,95 \%$ CI $[0.90,1.90], p=0.15$; Figure 5) and incidence of miscarriage (No prior IVF group' RR 1.23, 95\% CI [0.82, 1.83], p $=0.32$; Recurrent IVF failure group' RR $0.91,95 \%$ CI $[0.74,1.13]$, $\mathrm{p}=0.39)$. The pooled analysis were not homogenous $(P<0.1)$

\section{SENSITIVITY ANALYSIS}

Statistically significant heterogeneity was detected in most of pooled outcomes. This heterogeneity was best resolved by excluding either Smit et al. or El-Toukhy, et al. Both trials reported statistically insignificant effect of hysteroscopy in pregnancy outcomes $^{[6,20]}$. 


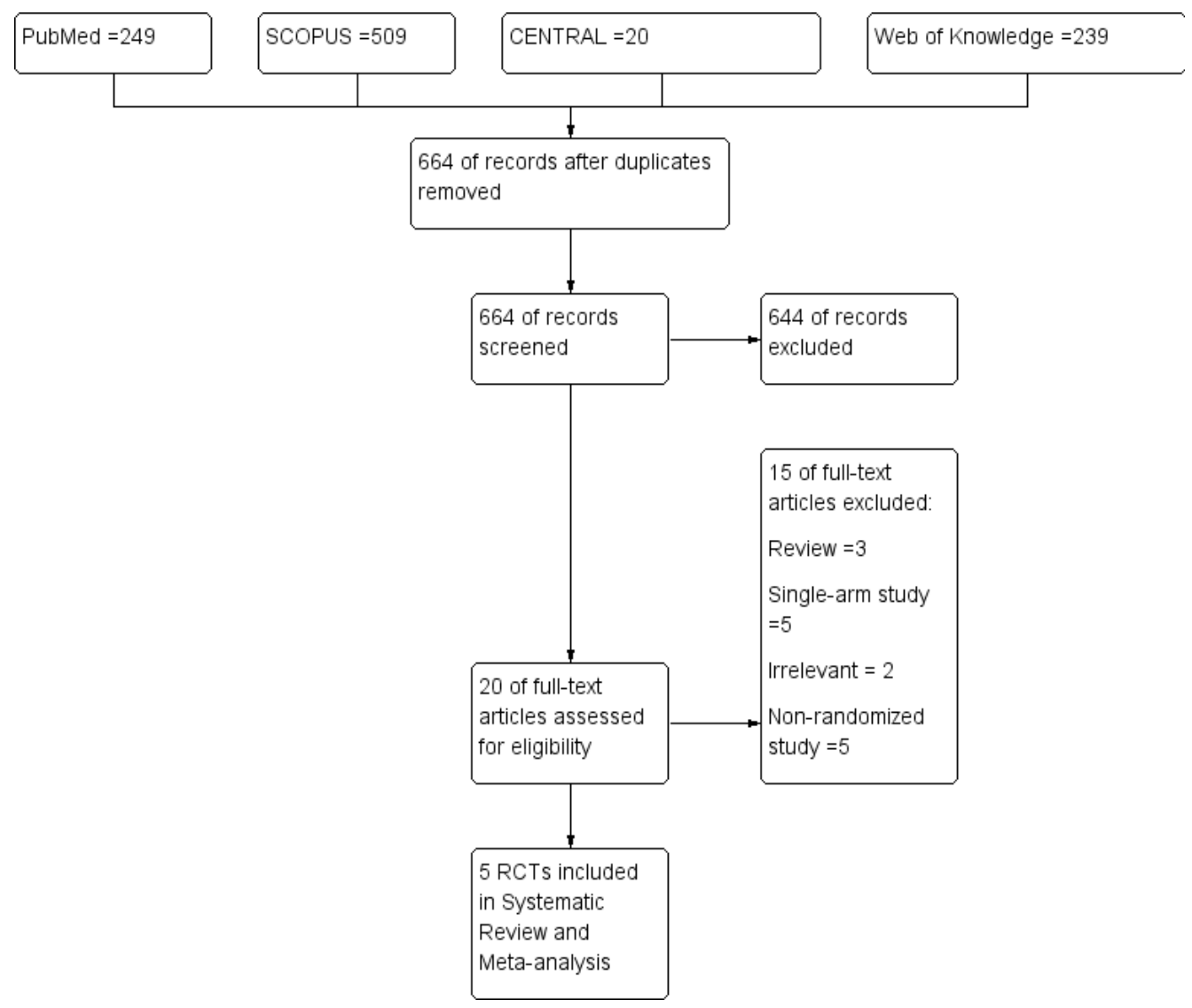

Fig. 1: Shows the PRISMA flow diagram of studies' screening and selection

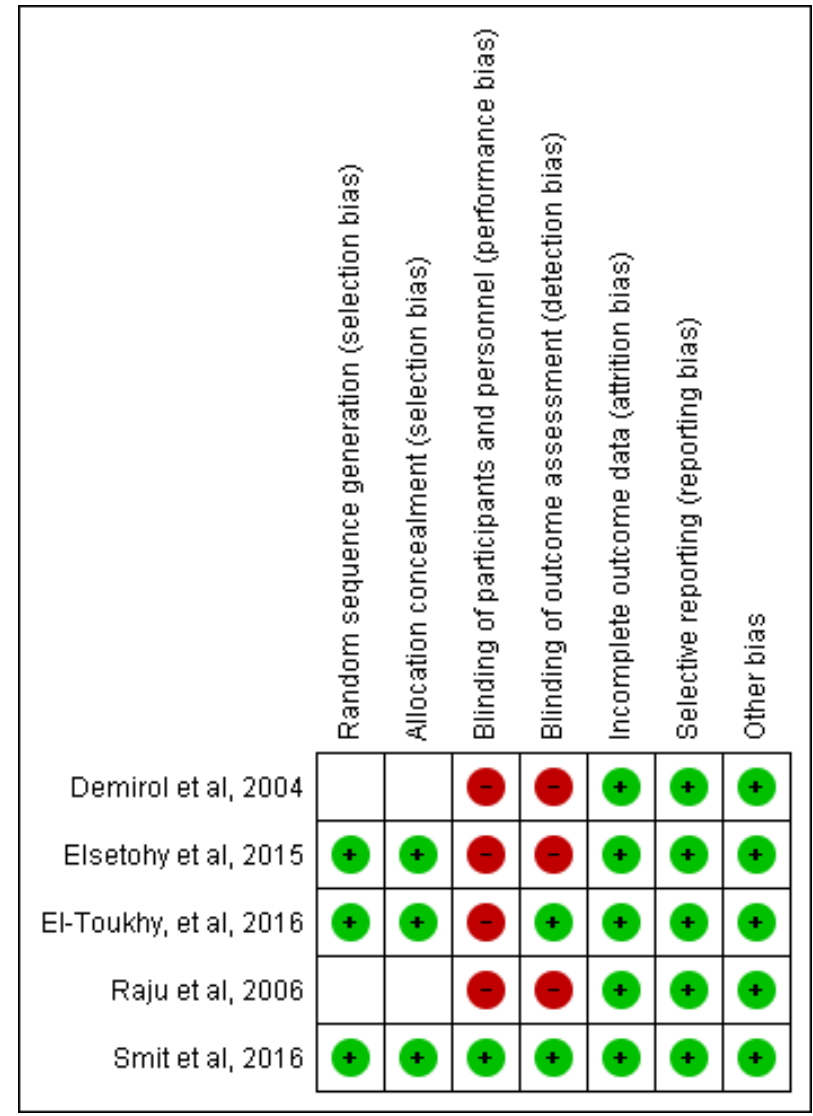

Fig. 2: Shows the risk of bias summary and risk of bias graph according to Cochrane Risk of Bias assessment tool 


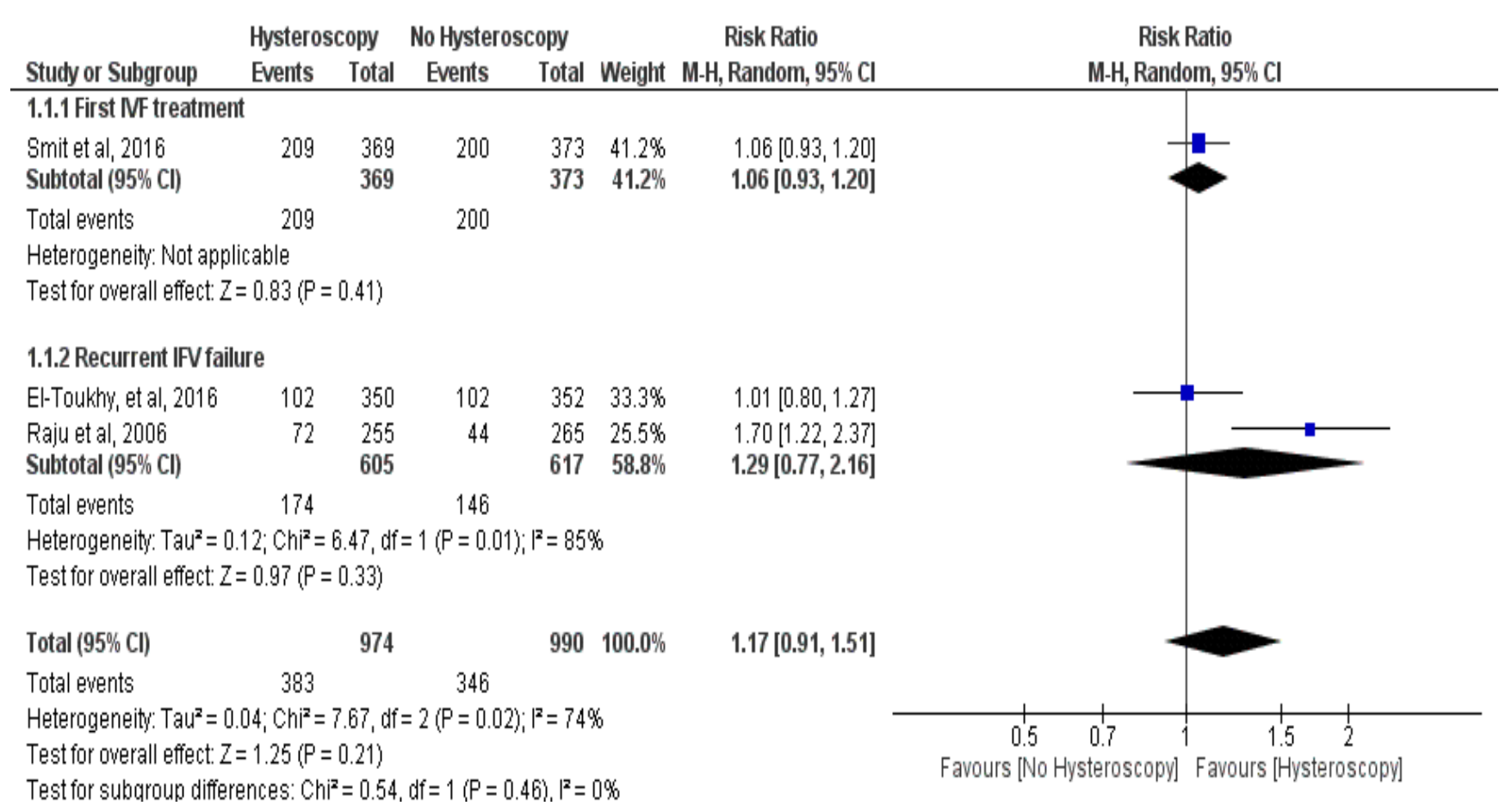

Fig. 3 : Shows forest plots of relative risk in a live birth from a pregnancy during the trial period. RR=Relative risk, M-H=Mantel-Haenzel, $\mathrm{CI}=$ confidence interval

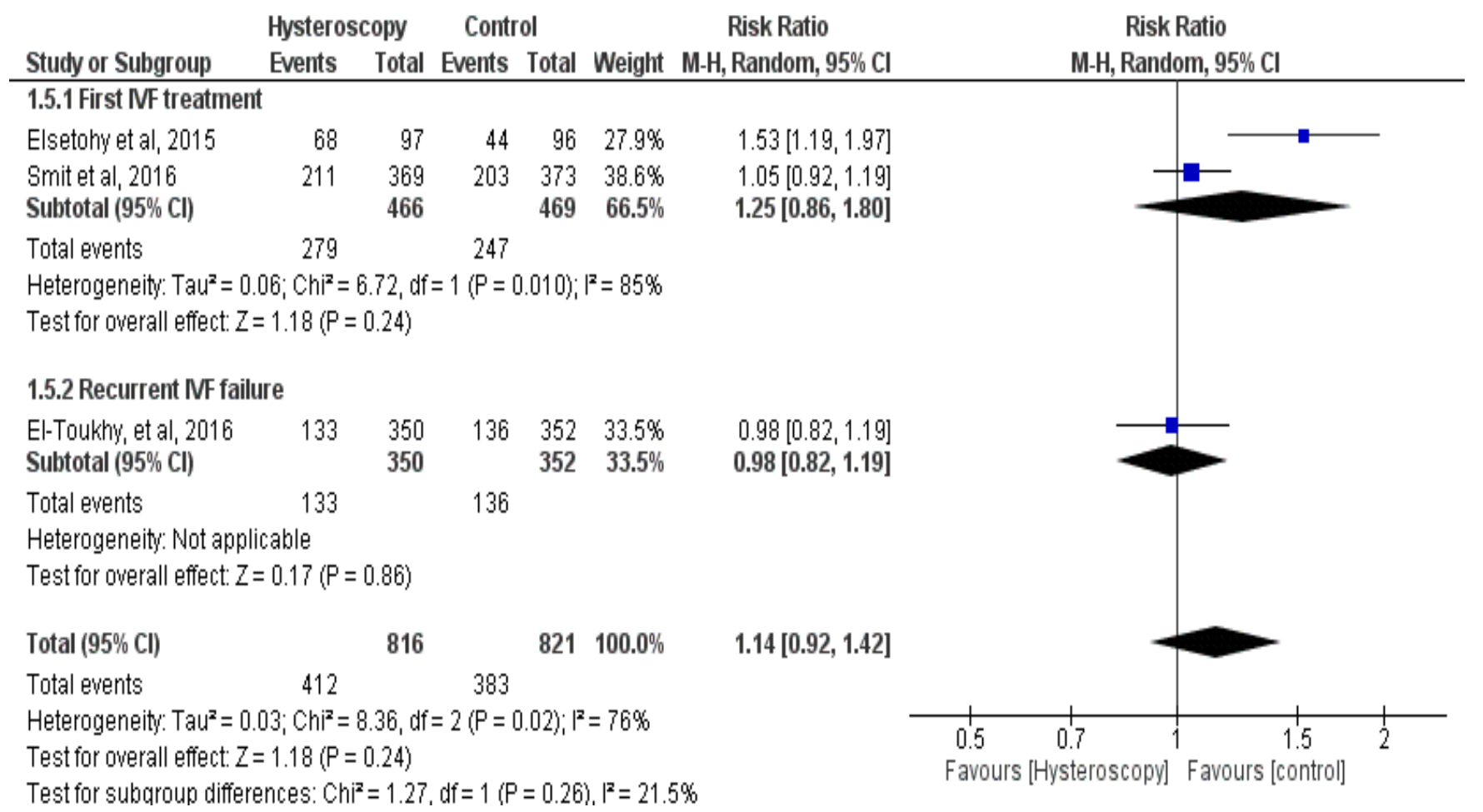

Fig. 4: Shows forest plots of relative risk in ongoing pregnancy during the trial period. RR=Relative risk, M-H=Mantel-Haenzel, CI=confidence interval 


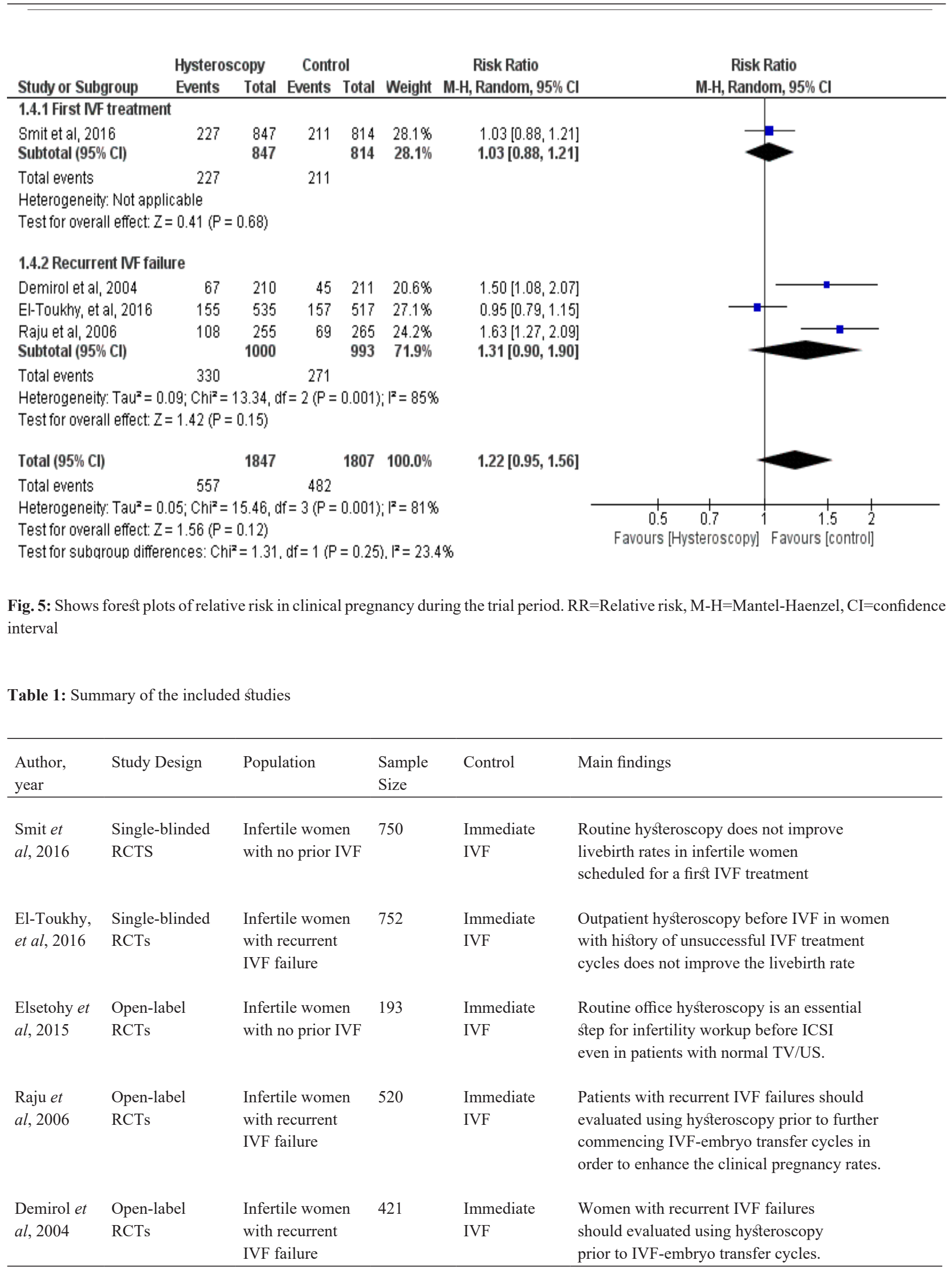




\section{DISCUSSION}

Uterine cavity abnormalities, as polyps, have been linked to a reduced chance of successful pregnancy among IVF treated women ${ }^{[15,22]}$ which lead to the hypothesis that hysteroscopy may improve pregnancy rates through removal of such abnormalities. The present meta-analysis shows that the current evidence is insufficient to support the routine use of hysteroscopy in both women scheduled for first IVF and women with recurrent IVF failure. Our pooled analysis showed that hysteroscopy did not improve live birth rates, ongoing pregnancy rates, clinical pregnancy rates, or reduced the incidence of miscarriage.

This result comes in concordance with the recent large two RCTs. This insight trial was an openlabel multicenter study that included 750 infertile women who underwent their first IVF treatment cycle, the study reported insignificant improvement in pregnancy outcomes among women who underwent hysteroscopy before $\operatorname{IVF}^{[20]}$. Similarly, TROPHY trial observed no difference in live birth rates among women with recurrent IVF failure who underwent hysteroscopy in comparison to women who scheduled for IVF directly ${ }^{[6]}$. In contrary, previous RCTs reported that hysteroscopy leads to more successful pregnancy outcome among women scheduled for first IVF treatment. However, these trials have many methodological limitations as small sample size, the quality of embryo transfer, and the singlecenter deign ${ }^{[4,7,17]}$. The observed heterogeneity in the published literature may be attributed to different inclusion criteria, two RCTs excluded women who had previous intrauterine surgery ${ }^{[6,20]}$, three trials included only women with normal trans-vaginal ultrasound (TVUS $)^{[6,7,20]}$, and all studies included women with different duration of infertility. Demirol et al. and Raju et al. did not report whether the pathologies they noted at hysteroscopy were suspected or not at TVUS, which did not allow to compare their results with trials who included women with normal TVUS findings. In addition, the fact that hysteroscopy was done by different gynecologists in different studies might be regarded as a limitation because studies on the diagnostic accuracy of hysteroscopy have shown that it is associated with a considerable degree of interobserver variability. ${ }^{[1,20]}$

Moreover, a meta-analysis by Pundir et al. reported a different results from our polled analysis by showing that hysteroscopy increases live birth rate among women scheduled for their first IVF treatment ${ }^{[16]}$. The mentioned meta-analysis included only one RCT and four non-randomized trials $(\mathrm{N}=3179$ participants $)$ which may increase the susceptibility to selection bias, and subsequently limit the quality of their results ${ }^{[9]}$. In contrary, our pooled analysis included only RCTs $(\mathrm{N}=2636$ patients $)$, that is least likely to be biased, with a transparent assessment of the quality of evidence.

\section{THE STRENGTHS AND THE LIMITATIONS} OF THE STUDY:

We performed this review according to PRISMA statement ${ }^{[14]}$. The strengths of the current meta-analysis comprise a comprehensive search of published clinical trials studies from multiple electronic databases. Furthermore, there was a transparent assessment of the quality of evidence.

The main limitation of this meta-analysis is the small number of included studies. Consequently, we cannot analysis the effect of uterine cavity abnormalities detected by TVUS using a subgroup analysis with a lot of data in details. Only one trial was blinded, which increase the risk of performance bias ${ }^{[6]}$.

\section{CONCLUSION}

In conclusion, the present meta-analysis shows that the current evidence is insufficient to support the routine use of hysteroscopy in both women scheduled for first IVF and women with recurrent IVF failure. Our pooled analysis showed that hysteroscopy did not improve live birth rates, ongoing pregnancy rates, clinical pregnancy rates, or reduced the incidence of miscarriage.

\section{ACKNOWLEDGMENT}

Ahmed Elgebaly has full access to all data in the study and takes responsibility for the integrity of presented information and accuracy of the data analysis.

Study concept and design:

Internet searching:.

Selection of studies:

Data extraction:

Quality assessment:

Data analysis:

Drafting the manuscript:

Revision and appraisal of the manuscript:

Proofreading the manuscript:

Study monitoring \& supervision: Ahmed Elgebaly

\section{CONFLICT OF INTEREST}

There are no conflicts of interest. 


\section{REFERENCES}

1. Aletebi, F., 2010. Hysteroscopy in women with implantation failures after in vitro fertilization: Findings and effect on subsequent pregnancy rates. Middle East Fertil. Soc. J. 15, 288-291. https://doi.org/10.1016/j.mefs.2010.06.008

2. Bouwmans, C.A.M., Lintsen, B.M.E., Eijkemans, M.J.C., Habbema, J.D.F., Braat, D.D.M., Hakkaart, L., 2008. A detailed cost analysis of in vitro fertilization and intracytoplasmic sperm injection treatment. Fertil. Steril. 89, 331-341. https://doi.org/10.1016/j. fertnstert.2007.03.003

3. Cenksoy, P., Ficicioglu, C., Yildirim, G., Yesiladali, M., 2013. Hysteroscopic findings in women with recurrent IVF failures and the effect of correction of hysteroscopic findings on subsequent pregnancy rates. Arch. Gynecol. Obstet. 287, 357-360. https://doi.org/10.1007/s00404-012-2627-5

4. Demirol, A., Gurgan, T., 2004. Effect of treatment of intrauterine pathologies with office hysteroscopy in patients with recurrent IVF failure. Reprod. Biomed. Online 8, 590-4. https://doi.org/10.1016/S147261108-(10)6483-X

5. Egger, M., Davey Smith, G., Schneider, M., Minder, C., 1997. Bias in meta-analysis detected by a simple, graphical test. BMJ 315, 629-634. https://doi.org/10.1136/bmj.316.7129.469

6. El-Toukhy, T., Campo, R., Khalaf, Y., Tabanelli, C., Gianaroli, L., Gordts, S.S., Gordts, S., Mestdagh, G., Mardesic, T., Voboril, J., Marchino, G.L., Benedetto, C., Al-Shawaf, T., Sabatini, L., Seed, P.T., Gergolet, M., Grimbizis, G., Harb, H., Coomarasamy, A., 2016. Hysteroscopy in recurrent in-vitro fertilisation failure (TROPHY): a multicentre, randomised controlled trial. Lancet 387, 2614-2621. https://doi.org/10.1016/S014000258-0(16)6736-

7. Elsetohy, K.A.A.A., Askalany, A.H., Hassan, M., Dawood, Z., 2015. Routine office hysteroscopy prior to ICSI vs. ICSI alone in patients with normal transvaginal ultrasound: a randomized controlled trial. Arch. Gynecol. Obstet. 291, 193-199. https://doi.org/10.1007/s004043397--014-z

8. Green, S., Higgins P., T., J., Alderson, P., Clarke, M., Mulrow D, C., Oxman D, A., 2011. Cochrane Handbook: Cochrane Reviews: Ch 8: Assessing risk of bias in included studies, in: Cochrane Handbook for: Systematic Reviews of Interventions. pp. 3-10.

9. Higgins, J.P., Green, S., 2008. Cochrane Handbook for Systematic Reviews of, The Cochrane Collaboration. https://doi.org/10.10029780470712184/

10. Kamel, R.M., 2010. Management of the infertile couple: an evidence- based protocol. Reprod. Biol. Endocrinol. 8, 21. https://doi.org/10.118621-8-7827-1477/

11. Kasius, J.C., Broekmans, F.J.M., Veersema, S., Eijkemans, M.J.C., van Santbrink, E.J.P., Devroey, P., Fauser, B.C.J.M., Fatemi, H.M., 2011. Observer agreement in the evaluation of the uterine cavity by hysteroscopy prior to in vitro fertilization. Hum. Reprod. 26, 801-807. https://doi.org/10.1093/humrep/der003

12. Kodaman, P.H., 2016. Hysteroscopic polypectomy for women undergoing IVF treatment: When is it necessary? Curr. Opin. Obstet. Gynecol. https://doi.org/10.1097/GCO.0000000000000277

13. Margalioth, E.J., Ben-Chetrit, A., Gal, M., EldarGeva, T., 2006. Investigation and treatment of repeated implantation failure following IVF-ET. Hum. Reprod. https://doi.org/10.1093/humrep/del305

14. Moher, D., Liberati, A., Tetzlaff, J., Altman, D.G., 2009. Preferred reporting items for systematic reviews and meta-analyses: the PRISMA statement. PLoS Med. 6, e1000097. https://doi.org/10.1371/journal.pmed.1000097

15. Pérez-Medina, T., Bajo-Arenas, J., Salazar, F., Redondo, T., Sanfrutos, L., Alvarez, P., Engels, V., 2005. Endometrial polyps and their implication in the pregnancy rates of patients undergoing intrauterine insemination: a prospective, randomized study. Hum. Reprod. 20, 1632-1635. https://doi.org/10.1093/humrep/deh822

16. Pundir, J., Pundir, V., Omanwa, K., Khalaf, Y., El-Toukhy, T., 2014. Hysteroscopy prior to the first IVF cycle: A systematic review and meta-analysis. Reprod. Biomed. Online. https://doi.org/10.1016/j.rbmo.2013.09.025

17. Rama Raju, G.A., Shashi Kumari, G., Krishna, K.M., Prakash, G.J., Madan, K., 2006. Assessment of uterine cavity by hysteroscopy in assisted reproduction 
programme and its influence on pregnancy outcome. Arch. Gynecol. Obstet. 274, 160-164. https://doi.org/10.1007/s00404-006-0174-7

18. Schmidt, L., 2006. Infertility and assisted reproduction in Denmark. Epidemiology and psychosocial consequences. Dan. Med. Bull. 53, 390-417. https://doi.org/DMB3808 [pii]

19. Smit, J.G., Kasius, J.C., Eijkemans, M.J.C., Koks, C.A.M., van Golde, R., Nap, A.W., Scheffer, G.J., Manger, P.A.P., Hoek, A., Schoot, B.C., van Heusden, A.M., Kuchenbecker, W.K.H., Perquin, D.A.M., Fleischer, K., Kaaijk, E.M., Sluijmer, A., Friederich, J., Dykgraaf, R.H.M., van Hooff, M., Louwe, L.A., Kwee, J., de Koning, C.H., Janssen, I.C.A.H., Mol, F., Mol, B.W.J., Broekmans, F.J.M., Torrance, H.L., 2016. Hysteroscopy before in-vitro fertilisation (inSIGHT): a multicentre, randomised controlled trial. Lancet (London, England) 387, 2622-2629. https://doi.org/10.1016/S0140-6736(16)00231-2

20. Smit, J.G., Kasius, J.C., Eijkemans, M.J.C., Veersema, S., Fatemi, H.M., Santbrink van, E.J.P., Campo, R., Broekmans, F.J.M., 2013. The international agreement study on the diagnosis of the septate uterus at office hysteroscopy in infertile patients. Fertil. Steril. 99, 2108-2113.e2. https://doi.org/10.1016/j.fertnstert.2013.02.027

21. Stewart, L.M., Holman, C.D.A.J., Hart, R.,
Finn, J., Mai, Q., Preen, D.B., 2011. How effective is in vitro fertilization, and how can it be improved? Fertil. Steril. 95, 1677-1683. https://doi.org/10.1016/j.fertnstert.2011.01.130

22. Taylor, E., Gomel, V., 2008. The uterus and fertility. Fertil. Steril https://doi.org/10.1016/j.fertnstert.2007.09.069

23. Terrin, N., Schmid, C.H., Lau, J., Olkin, I., 2003. Adjusting for publication bias in the presence of heterogeneity. Stat. Med. 22, 2113-2126. https://doi.org/10.1002/sim.1461

24. Varasteh, N.N., Neuwirth, R.S., Levin, B., Keltz, M.D., 1999. Pregnancy rates after hysteroscopic polypectomy and myomectomy in infertile women. Obstet. Gynecol. 94, 168-171. https://doi.org/10.1016/S0029-7844(99)00278-1

25. Wade, J.J., MacLachlan, V., Kovacs, G., 2015. The success rate of IVF has significantly improved over the last decade. Aust. New Zeal. J. Obstet. Gynaecol. 55, 473-476. https://doi.org/10.1111/ajo.12356

26. Zhao, Y., Brezina, P., Hsu, C.C., Garcia, J., Brinsden, P.R., Wallach, E., 2011. In vitro fertilization: Four decades of reflections and promises. Biochim. Biophys. Acta - Gen. Subj. https://doi.org/10.1016/j.bbagen.2011.05.001. 